American Journal of Applied Sciences 2 (8): 1270-1273, 2005

ISSN 1546-9239

(c) 2005 Science Publications

\title{
Large Eddy Simulation of an Ammonia Jet
}

\author{
${ }^{1}$ M. Salinas-Vazquez, ${ }^{1}$ W. Vicente, ${ }^{3}$ A. Espinosa and ${ }^{2}$ E. Barrios \\ ${ }^{1}$ Instituto de Ingeniería, ${ }^{2}$ Facultad de Ingeniería, UNAM, 04510 México D.F., Mexico \\ ${ }^{3}$ Instituto Mexicano de Tecnología del agua, 62565 Jiutepec Mor., Mexico
}

\begin{abstract}
A compressible jet flow of ammonia was studied through a series of Large Eddy Simulations. Simulations were focused in the zone where the inertial effects are much bigger than the gravitational ones. The ammonia jet is discharged into an air medium and the gases are treated as a two non-reactive ideal gas mixture. Different simulations were performed, going from the use of constant transport properties to the use of kinetic theory of gases relations for calculating them as a function of the resolved variables. The main goal of the present study is to investigate the effect of varying density as well as variable transport properties on the turbulent flow behavior.
\end{abstract}

Key words: LES, compressible flow, gas mixing, jet, ammonia

\section{INTRODUCTION}

The study of accidental atmospheric jet releases of toxic gases together with its subsequent dispersion and the mixing of gases in combustion or in industrial processes are of considerable engineering interest. If the mixing gases have different densities, distinct effects on the global and turbulent variables are observed ${ }^{[1,2]}$. When the transport properties are added to the study, they can also influence the flow. In this work, an isothermal ammonia jet discharged to an air atmosphere was studied. Both substances are considered as nonreactive ideal gases.

In general, the study of a jet with variable density can be divided into three different regions: the pure inertial zone, the buoyant plume zone and intermediate zone, where both, inertial and buoyant forces, are important. The present simulations are focused in the first zone, where inertial forces are dominant. This zone comprises many nozzle diameter lengths and goes from the jet nozzle to $x_{\text {inertial }} / D<F^{1 / 2} r_{\rho}^{1 / 4}(x / D)^{[3]}$; where $D$ is the jet nozzle diameter, $\mathrm{Fr}$ is the Froude number, $r_{\rho}$ is the ammonia/air density ratio and $x$ the longitudinal direction.

Because it is in the inertial zone where the turbulence development is produced, a study of the fluctuating variables and its correlations is performed. Different Large Eddy Simulations were considering different approaches for density and for transport properties. The main goal of this work is to investigate the effect of variable density and transport properties in the turbulence development and intensity through the inertial zone. For this purpose, a statistical study and instantaneous visualizations of the flow were performed.

\section{NUMERICAL CONSIDERATIONS}

The Large Eddy Simulation filtered governing equations were solved in generalized coordinates by mean of an extension of the fully explicit Mac-Cormack scheme, fourth order in space and second order in time $^{[4]}$. The sub-grid scale tensor was modeled through the classical eddy-viscosity assumption, computed from a subgrid-scale model, wherein the selective structure function model was applied ${ }^{[5]}$. Compressible equations were normalized using the following reference dimensions: relative inlet jet velocity $U_{j}$, jet density $\rho_{j}$, jet diameter $D$ and jet temperature $T_{j}$. The inlet and outlet boundary conditions ( $i x=1$ and $i x=n x$, respectively) were based in the Poinset \& Lele boundary conditions ${ }^{[6]}$. A sponge layer was used in the outflow zone in order to attenuate outflow disturbances $^{[7]}$. Free boundaries were imposed at $i y=1$ and $i y=n y^{[8]}$. Periodic boundary condition was imposed in the spanwise direction, $z$, (Fig. 1).

For the cases where the viscosity and the thermal conductivity are function of solving variables, Southerland law was used ${ }^{[9]}$. The coefficient of mass diffusivity was obtained by the expression for binarydiffusion coefficient between the two species, air/ammonia, of Chapman and Cowling ${ }^{[10]}$.

Computational domains and flow configuration: Dimensions of all computational domains are: $22 \mathrm{D} \mathrm{X}$ $12 \mathrm{D} \times 6 \mathrm{D}$ in the $x$ (streamwise), $y$ (normal) and $z$ (spanwise) directions, respectively. In order to correctly simulate the near shear layer regions, a non uniform (orthogonal) grid with hyperbolic-tangent stretching ${ }^{[11]}$ is used in the $y$ direction. The spanwise length has also shown that it can ensure the correct representation of the longest turbulent structures present within the flow.

The computational grid consists of 120 X 109 X 60 nodes along the $x, y$ and $z$ directions, respectively.

Corresponding Author: M. Salinas-Vazquez, Instituto de Ingeniería, UNAM, 04510 México D.F., Mexico 
Simulations were performed at a Reynolds and Mach number of $\mathrm{Re}=4000$ and $\mathrm{M}=0.3$ based on the reference dimensions.

A velocity profile was prescribed at the inlet at each time step. This inlet velocity is based on a hyperbolic tangent profile. A three-component fluctuating velocity field (10\% of the maximal inlet velocity) was superimposed within the mean velocity shear layer region ${ }^{[12,13]}$. For all simulations, the maximal and minimal (co-flow) velocity are 1.1 and 0.1 $\mathrm{U}_{\mathrm{j}}$. The co-flow is very similar the one used by the Silva and Métais ${ }^{[13]}$ and Stanley et al. ${ }^{[12]}$. The inlet temperature is constant and the inlet density is a function of the gas mixture.

Four simulations, air/ammonia jet, were performed. The first one, LES_AIR, is a validation case where jet and atmospheric gases are air. The second one, LES_D, is an ammonia jet in air atmosphere considering just the different gas density in the mixture, transport properties is treated as constant and equal to those of the air at 300 $\mathrm{K}$. In a third case, LES_V, an ammonia jet in air atmosphere was simulated considering different gas density in the mixture, viscosity is a function of the temperature and other transport properties are computed through constant Prandtl and Schimdt numbers. And finally, LES_C is an ammonia jet in air atmosphere considering different densities and transport properties of both gases in the mixture; transport properties are treated as function of temperature and/or pressure. For thermal conductivity and viscosity, the Wilke equation ${ }^{[9]}$ is used for obtaining the mixture value.

\section{RESULTS}

In order to validate our numerical procedure and the subgrid scale model, an isothermal air jet was simulated, LES_AIR. The results of these LES were compared with the DNS of Stanley et al. ${ }^{[12]}$ and the experimental jet study of Ramparian and Chandrasekhara ${ }^{[14]}$, Gutmark and Wygnansky ${ }^{[15]}$ and Thomas and $\mathrm{Chu}^{[16]}$. Figure 2a, presents a comparison of the jet width, $\delta_{u}$, defined as the distance from the jet centerline to the point at which the mean streamwise velocity excess is half of the centerline value, $\mathrm{U}_{\mathrm{C}}$. The self-similar region has a linear relation and all four simulations attain this zone at almost the same point, $x / D(5$. The growth rates values of the jets agree very well with DNS and experimental results. LES_AIR and LES_D present the lower growth rates. In the present simulations the self-similar regime is attained later, in Fig. 2a, $\delta_{u}$ profile was shifted down a distance $\Delta x$ for a better comparison; this retard is due, mainly, to the boundary inlet conditions sensitivity. The normal velocity component, $V$, at the self-similar region $x / D \approx 17$, presents a plateau at the shear layer zone. Differences are observed in Fig. $2 b$ between the simulations, observing the highest values for LES_C. However, the streamwise velocity, not presented here, does not show these differences.

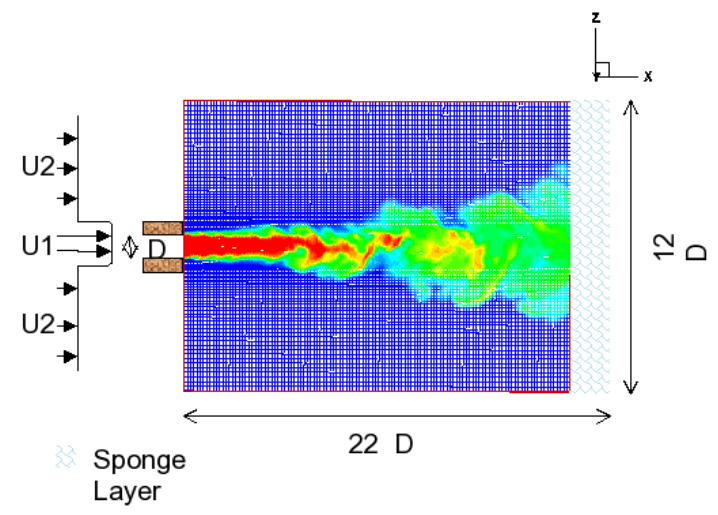

Fig. 1: Jet configuration

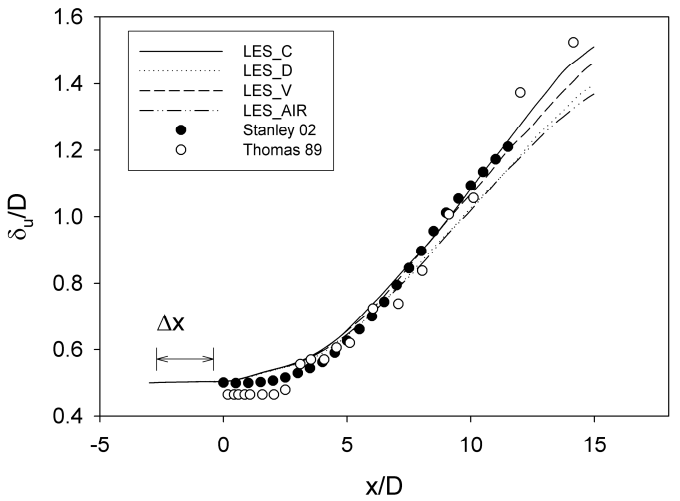

(a)

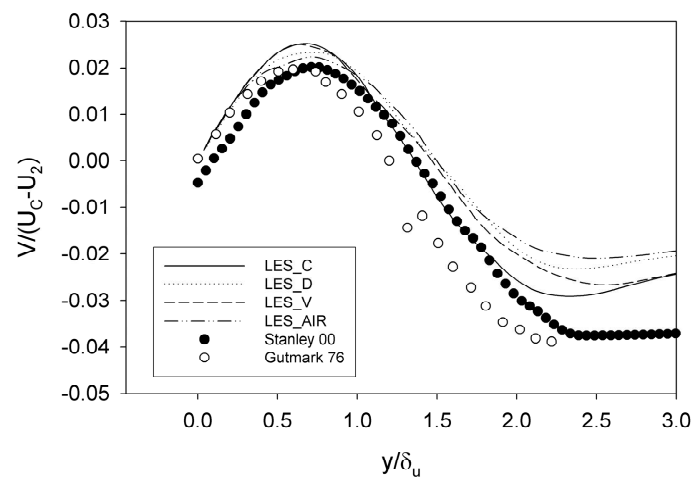

(b)

Fig. 2: a) Jet width, $\delta u$, in function of the streamwise direction, at the centerline $y / D=6 ; b)$ mean normal velocity component normalized by the centerline velocity $\left(\mathrm{U}_{\mathrm{C}}-\mathrm{U}_{2}\right)$, in function of the normal direction $\mathrm{y} / \delta \mathrm{u}$, at $\mathrm{x} / \mathrm{D} \approx 17$

Figure 3 shows the comparison of the fluctuating velocity rms (root mean square) in function of the direction normal to the flow at the self-similar region $x / D \approx 17$. The $y$ coordinate is normalized by $\delta_{u}$. The four LES profiles have a similar behavior and differences are observed mainly in the shear layer jet zone, where all profiles have a plateau. The LES_V and LES_C developed the maximal values, which are more than 10\% higher for $v^{\prime}{ }_{r m s}$ and around 5\% higher for $u^{\prime}{ }_{r m s}$ compared with the other simulations. 


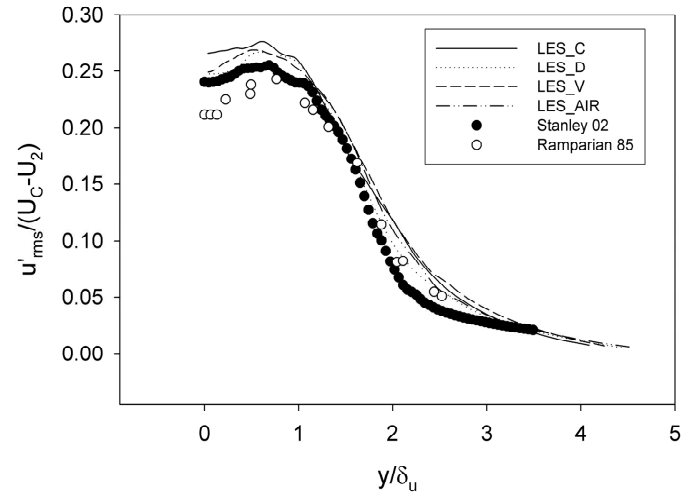

(a)

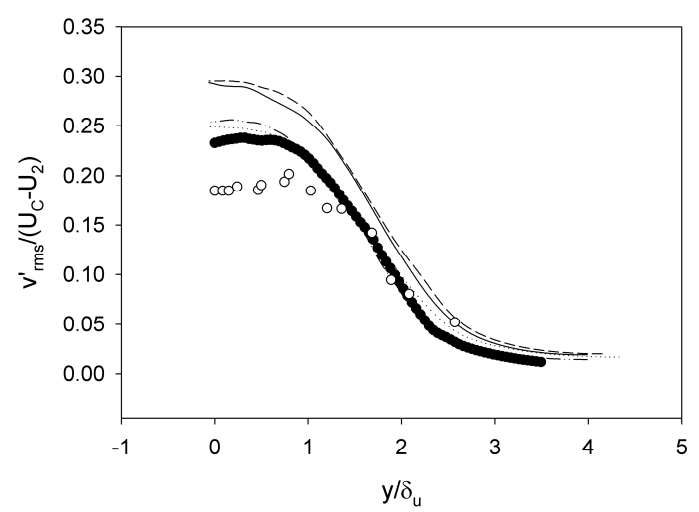

(b)

Fig. 3: a) rms of the fluctuating streamwise velocity, $\mathrm{u}_{\text {rms }}$, in function of the normal direction $\mathrm{y} / \mathrm{\delta u}$, at $\mathrm{x} / \mathrm{D} \approx 17 ; \quad$ b) $\mathrm{rms}$ of the fluctuating normal velocity, $v$ 'rms, by the function of the normal to the flow direction $\mathrm{y} / \delta \mathrm{u}$, at $\mathrm{x} / \mathrm{D} \approx 17$

Differences in density and in the modeling of transport properties seem to be linked with a higher turbulent activity in the normal direction, $y$. Although a comparison with experimental data of binary gas jets was not performed, results obtained in this work are in concordance with the behavior observed in this kind of flows $^{[1,2]}$.

Figure 4 shows the thermodynamic variable behavior, it presents the mean and the rms fluctuating values of the center line density and temperature as a function of the streamwise direction, $x$. The mean density in LES_D presents a lower development, indicating a lower mixing level in this jet. This simulation, as it was mentioned in the preceding section, does not consider variations in the transport properties and its poor development is directly related to it. In the mean temperature profiles, the LES_C presents an enhancement of the temperature of $\approx 4 \%$ in comparison with two other simulations and its value decreases and tends to the other LES values as $x$ increases.

The LES_C rms profiles present an earlier development, attaining faster the maximum plateau zone value at $x / D \approx 6$. 5. However, its $T^{\prime}{ }_{r m s}$ profile has lower maximal values.

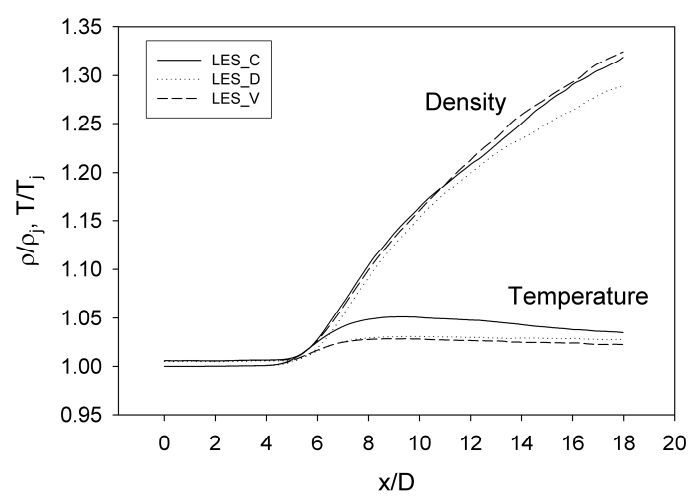

(a)

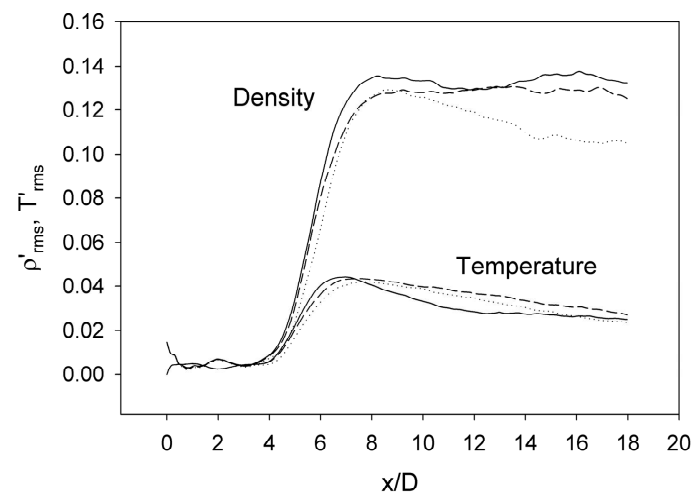

(b)

Fig. 4:a) Mean and rms values of the fluctuating density in function of the streamwise direction, at the centerline $y / D=6 ; b$ ) Mean and rms values of the fluctuating temperature in function of the streamwise direction, at the centerline $y / D=6$

It is not possible to prove with the present results, but it can be supposed that this temperature behavior relates to the appropriate computation of the thermal conductivity from the empirical Sutherland law. For LES_C jet simulation, in general, it was observed a more important turbulent activity and an earlier turbulent development.

It is common incompressible flow simulations to compute the thermal conductivity and binary mass diffusion coefficient from the viscosity, as in the LES_V case, considering constant values of the Prandtl and Schmidt number. In Fig. 5 the instantaneous contours of viscosity, thermal conductivity and binary mass ammonia/air diffusion coefficient are presented in the LES_C case. Although all three properties follow a similar pattern (they are functions of temperature and/or pressure), no relation is observed between the three property values; that is because every property was evaluated in an independent way. In spite of a higher computational cost for the LES_C case, in free turbulent binary gas mixing, this approach seems to be the better option for studying every variable of the problem. 


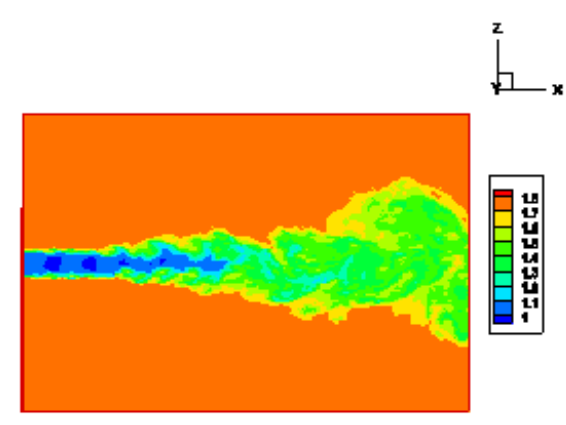

(a)

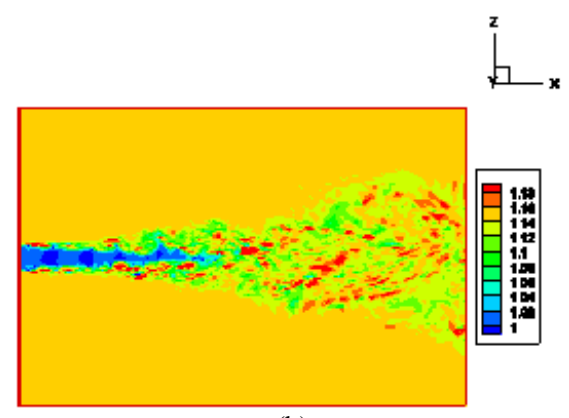

(b)

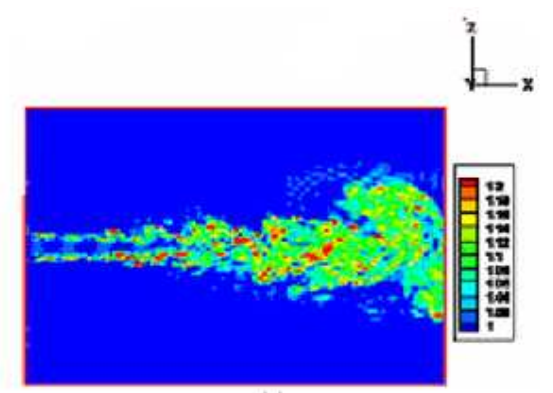

(c)

Fig. 5:Instantaneous contours, LES_C: a) viscosity, $\mu / \mu_{0} ;$ b) thermal conductivity, $\bar{k} / \mathrm{k}_{0}$ and c) binary mass diffusion coefficient, $\mathrm{C} / \mathrm{C}_{0}$. Properties are normalized by its value at the reference state, $T_{j}$ and $\mathrm{P}_{\mathrm{j}}$

\section{CONCLUSION}

In this work, different ways of modeling an ideal binary gas mixing jet was presented. The modeling of the transport properties was based on kinetic gas theory and on empirical relations like the Sutherland law. Although the turbulent quantities show the higher differences, it was shown that the mean values could present important differences also due, mainly, to the increase/decrease of the mixing rates. When the transport properties of the two gases are considerably different $\left(\mu_{\text {aire }} / \mu_{\text {ammonia }} \approx 1.8\right)$, the mixture transport properties must be computed in a correct way. The use of the method of constant Prandtl and Schmidt numbers produce an error of more than $50 \%$ with respect to the thermal conductivity and binary mass coefficient values computed in the LES_C case; this error is reflected in differences not only in turbulent variable, but also in the mean ones. From precedent results, it is possible to conclude in the importance of the correct modeling of mixing gas and its influence over the simulation results.

\section{ACKNOWLEDGEMENTS}

This work was supported by the Consejo de Ciencia y Tecnología, México. Under project CONACyT-SEMARNAT-2002-C01-148.

\section{REFERENCES}

1. Sanders, J.P.H., B. Sarh and I. Gökalp, 1996. Variable density effects in axisymmetric isothermal turbulent jet: A comparison between a first-ans a second-order turbulence model. Int. J. Heat Mass Transfer, 40: 823-842.

2. Chassaing, P., G. Harran and L. Joly, 1994. Density fluctuation correlations in free turbulent binary mixing. J. Fluid Mech., 279: 239-278.

3. Chen, C.J. and W. Rodi, 1980. Vertical Turbulent Bouyant Jets, A Review of Experimental Data. Pergamon Press, Oxford.

4. Gottlieb, D. and E. Turkel, 1976. Dissipative two four methods for time-dependent problems. Math. Comput., 30: 703-723.

5. Salinas Vázquez, M. and O. Métais, 2002. Large eddy simulation of the turbulent flow through a heated square duct. J. Fluid Mech., 453: 201-238.

6. Poinsot, T. and S. Lele, 1992. Boundary conditions for direct simulations of compressible viscous flows. J. Comp. Phys., 101: 104-129.

7. Sandhu, H.S. and N.D. Sandham, 1994. Boundary conditions for spatially growing compressible shear layer. Report QMW-EP-1100, Faculty of Engineering, Queen Mary and Westfield College, University of London.

8. Thompson, K.W., 1990. Time dependent boundary conditions for hyperbolic systems. J. Comp. Phys., 89: 439-461.

9. White, F.M., 1991. Viscous Flow. McGraw Hill.

10. Maitland, G.C., M. Rigby, E.F. Smith and W. Wakeham, 1981. Intermolecular Forces: Their Origin and Determination. Clarendon Press, Oxford.

11. Le, H., P. Moin and J. Kim, 1997. Direct numerical simulation of turbulent flow over a backwardfacing step. J. Fluid Mech., 330: 349-374.

12. Satanley, S.A., S. Sarkar and J.P. Mellado. A study of the flow-field evolution and mixing in a planar turbulent jet using direct numerical simulation. J. Fluid Mech., 450: 377-407.

13. da Silva, C.B. and O. Metais, 2002. On the influence of coherent structures upon interscale interactions in turbulent plane jets. J. Fluid Mech., 473: 103-145.

14. Ramparian, B.R. and M.S. Chandrasekhara, 1985. LDA measurements in plane turbulent jets. Trans. ASME: J. Fluids Engg. 107: 264-271.

15. Gutmark, E. and I. Gygnanky, 1976. The planar turbulent jet. J. Fluid Mech., 73: 465-495.

16. Thomas, F.O. and H.C. Chu, 1989. An experimental investigation of the transition of the planar jet: Subharmonic suppression and upstream feedback. Phys. Physics, 1: 1566-1587. 